\title{
Design \& Development of IoT Based Rehabilitation Outdoor Landscape for Gait Phase Recognition
}

\author{
A Gaddam, T Wilkin, M Angelova \\ School of Information Technology, \\ Deakin University, Burwood, \\ Victoria, Australia \\ Email: Anuroop.Gaddam@deakin.edu.au
}

\author{
A Valera \\ School of Engineering \& \\ Computer Sciences, \\ Victoria University of Wellington, \\ Wellington, New Zealand \\ Email: Alvin.Valera@vuw.ac.nz
}

\author{
J McIntosh, B Marques \\ School of Architecture, \\ Victoria University of Wellington, \\ Wellington, New Zealand \\ Email: Jacqueline.Mcintosh@vuw.ac.nz
}

\begin{abstract}
Over a few decades, there is a steady accretion of life expectancy in many countries. Significant advances in modern healthcare technologies, medicines and overall health care awareness gave many to lead a prolonged healthy life. Over the past few years, there has been a huge demand for unobtrusive health monitoring systems from both medical professionals and the general populace to use these technologies for understanding the general health well being in order to improve life longevity. However, most of the available technologies for health monitoring are difficult for the general populace to access and most of the developed health monitoring systems are either deployed in a controlled environment or in healthcare facilities, thus severely limiting the ease of access to many, especially the elderly people. For effective health management of elderly people, evidence-based decision making, continuously monitoring the health status of the elderly demographic is paramount. In order to address these issues, we developed an innovative smart IoT based outdoor health monitoring system. The proposed system collects information about the mobility, posture, and overall gait of the person in an easy to access public outdoor setting such as parks, supermarkets etc. It is quite evident from multiple studies, that analysing the gait can be a useful tool in early detection of the declining health of individuals, as mobility is a key factor in any individual's well being. Also, detecting gait disorders earlier will allow for functional relief to the health care system with the possibility of reducing the number and severity of gait disorders through early intervention.
\end{abstract}

Index Terms - force sensor, internet of things, medical assistance, health informatics, non-intrusive monitoring, therapeutic outdoor landscapes, gait analysis, surface sensor

\section{INTRODUCTION}

The global population of those people aged 65 years old and above is expected to grow rapidly, by the year 2050 it is expected to be 1.5 billion people around the world [1]. This would create a tremendous strain on the existing health care services because of an increased dependency on the health care services by the elderly population. Many people are living longer because of advances in medical sciences and tendency of individuals to have a healthy lifestyle [2]. However, any physical or mental impairments that are common in elderly population could lead to many growing risks of health issues like dementia, loss of hearing-vision, reduced mobility and common cognitive disorders. As the ageing population proceeds to outpaced the existing health care services globally, there is a immense necessity for a paradigm shift in thinking about health services [2] and the necessity to shift from reactive health care delivery to preventive health care delivery [3], [4]. Using smart wireless sensors in conjunction with the Internet of Things enables us to develop a smart platform for combining bio-metric data with health services through eHealth, e-Care technologies. Information and communication technology (ICT) at its present capacity can now have an ability in determining who provides care, where, what, how, when health care services are provided [5], [6]. It is evident that the individual well-being and health of elderly people improve when they engage in physical activity mainly in outdoor locations [7]. The World Health Organisation (WHO) supports the idea of creating and using outdoor areas that can engage elderly people physically in their environment [1]. Coupled with this, there is an ever-increasing recent trend of age-specific therapeutic outdoor spaces around the world, in an attempt to decrease, overcome the possible old age-related ailments like loss of cognitive function, physical impairment and loss of mobility [8]. There is an immense need to evaluate the relevance and usage of assistive technologies in a therapeutic outdoor environment. There is also a need to develop smart systems that are able to collect and combine the bio-metric information with Electronic Health Record (EHR) services via the Internet of Things that are suitable for such outdoor landscapes.

Even though there are outdoor interactive spaces especially for older persons in some parts of the world, there is inadequate research exploring the use of current technologies and the design issues that actually determine what targeted interactive outdoor areas for aged persons actually should look like, whereabouts in our communities they could be placed, what are the technological issues that needs to be addressed and what sort of factors should be acknowledged within design schema [1], [9]. Therefore, currently, there is inadequate knowledge on addressing how technologies like IoT and smart sensors can be used in interactive outdoor landscapes in context to know how older persons health should best be addressed through engagement with interactive outdoor landscapes. While it is quite evident there is a growing trend for the incorporation of outdoor workout equipment into 
urban parks and walkways, there is a need to investigate how integrating technologies like IoT, sensors into the outdoor equipment can benefit its users and help aid current e-health management systems.

This paper seeks to contribute to this knowledge gap and presents a novel IoT based sensing systems, by informing on the appropriateness of various outdoor exercise strategies effective at supporting elderly health, well-being and also analyse, measure the gait, mobility. Finally, this paper ends with major findings, conclusions and discussions for future research.

\section{RELATED WORKS}

For effective health management of elderly people, evidence-based decision making, continuous monitoring the health status of the elderly demographic there is a need to develop a reliable and robust system that is intelligent to providing timely health status information [1]. A recent trend of increased demand for health information coupled with the availability of outdoor space provides a new opportunity to non-intrusively monitor aged people and provide interactive feedback on any age-related health issues. Wireless sensors embedded in the outdoor spaces does this effective measurement and communicate the captured pivotal data for further processing. To build smart open space to support elderly people in an outdoor environment the ambient intelligence and effective algorithms play an important role [10]. In [8], the authors review and identify sensor technologys potential to assess the functionality of older adults in outdoor spaces and finds lacking in the area of sensor used to detect frailty in elderly in outdoor spaces. There are many different sensing systems that can collect data for gait analysis and they are generally divided into two main categories of sensors: wearable and non-wearable. Most of the research that has been done in this area of monitor mobility and gait of elderly people is done by using wireless sensors in an indoor setting and/or using wearable sensors [11], [12]. However, recent study by Zhang et.al. attempt to use marker-free video analysis to determine abnormalities in gait. This uses software that isolates the patients silhouette and extracts the frequency of the gait for display in graphical forms [13]. In [14], presents a functional tool for gait abnormality detection that uses video analysis through automatic marker tracking. In [15], Heikkil et. al. designed an intelligent furniture using a network of wireless sensors which can be used in an outdoor space for tracking residents posture and detection of abnormal living patterns.

Research has shown that using multiple wearable sensors can provide more rich and accurate data [16]. However, this may only be relevant when performing gait analysis in a clinical area or in a laboratory. It may not be practical if the intention is to collect data about gait passively and frequently. Furthermore, acceptance from the older adults may also be a challenge. Some non-wearable sensors can also be costly and difficult to set up. For example, in a study conducted by Cedervall et.al. an optical motion capture system was used for three dimensional gait analysis with eighteen reflecting markers applied on defined anatomical landmarks [17]. The scalability of this set up when applied to a population of older adults can be costly, will take time to set up, and require an expert to install the markers. A similar way of capturing a persons gait that is gaining popularity in the recent years due to its simplicity to set up and low cost is the use of Microsofts Kinect Scanner [18].

Several studies has shown that it is able to detect gait and can be a useful tool in early detection of gait abnormalities. An embedded pressure sensor may provide more useful data in analysing foot pressure in relation to a persons gait [19]. However, there is no sufficient research done about using resistive pressure sensors alone in performing gait analysis [20]. One such study has used a Kistler force plate embedded along a walkway to measure the ground reaction forces and movement of centre of pressure [21]. In [22], a focus group study involving thirteen elderly conducted to know the general attitudes. The study concludes that the attitudes towards wearable sensors were not as positive as compared to attitudes toward embedded sensors. Participants pointed out issues such as the size of the sensor, the cost, and having to remember to wear it. Some non-wearable sensors can also be costly and difficult to set up. For example, in a study conducted by Cedervall et.al [23] an optical motion capture system was used for three dimensional gait analysis with eighteen reflecting markers applied on defined anatomical landmarks. Most research conducted have used wearable sensors to measure gait. There is however limited studies that involved non wearable sensors for gait analysis, particularly embedded sensor technologies. Some research has also shown that changes in gait could occur in the early stages of disease and can be useful in early diagnosis. Particular interest has been noted in the area of predicting future disability among older adults, specifically of Parkinsons disease. In this regard it is vital to be able to capture a persons gait, not only in the most accurate way possible, but also in multiple instances at regular intervals. This could enable early diagnosis of diseases and their complications possibly allowing for early treatment and more successful intervention. It is, therefore, apparent that there is an increasing demand for therapeutic landscapes to rekindle connections to smart cities around the world.

Also expanding the use and the effectiveness of these existing resources by incorporating technologies like IoT, smart embedded sensors can enhance these spaces for additional health benefits within the older population. This approach is of critical importance for elderly people's physical, mental and general social well-being. Currently, there is limited research that is done to understand, how the elderly people health should best be monitored, addressed through engagement with IoT based interactive smart outdoor landscapes. Also seldom adequate research has been done to try and apply a reasoner to generate new information and to expand it into the context of the using IoT's for outdoor therapeutic public spaces within smart cities. 


\section{Methodology}

The quantification of key data about an individual's posture and mobility, such as balance, gait and foot-ground interactions are the important characteristics for evaluating the quality of life of the people with any ongoing mobility issues, such as elderly [24]. Some of the chronic disorders faced my elderly, like arthritis, diabetic foot and neuro-degenerative conditions may result in reduced mobility thereby affecting the physical activity of the persons that, if not monitored or checked on time, can swiftly jeopardise the autonomy of the person [25].

A framework was designed and categorised in two levels; hardware prototype development and then followed by testing and programming logic. At the prototype development and testing, novel procedures were taken to develop the sensing unit, outdoor equipment that is needed to be positioned to acquire multi-movement and multi-event sensory data. The smart sensors embedded within the outdoor public equipment collects the semi-passive data about its users and then can be processed to extract the information about any deterioration, health anomalies of its users, especially the elderly and inform it to the health care professionals. An IoT paradigm based smart outdoor system with embedded sensors is developed and the overview of the system architecture is shown in Figure 1.

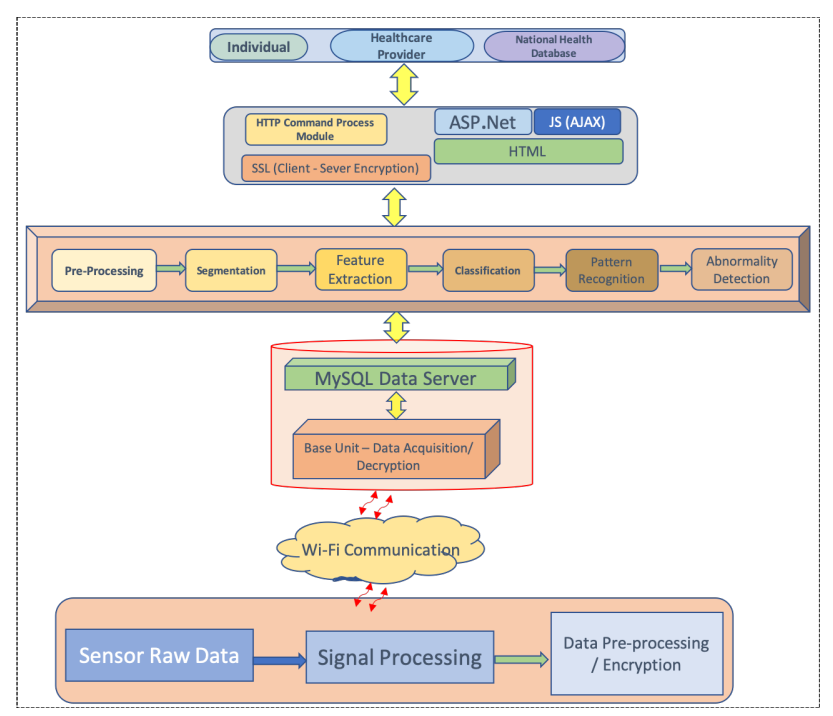

Fig. 1. System architecture of the IoT based Outdoor Therapeutic System.

The system consists of two main components: a smart sensory bench and a smart sensory path. The bench and path concepts propose that users would be given a Near-field communication (NFC) based identification card, where they could tag onto equipment, complete sixty seconds set of sit-to-stand exercises on the bench and walk to the opposite end of the smart path. During these exercises sensors from each system would collect pressure and touch data, which would then be transmitted to a database, processed and presented on a website that is viewed by the health care professionals. A simplified version of the data could also be viewed by the user on a mobile phone to offer feedback and reinforcement of the users progress. The primary benefits of this system are two-fold: the users will receive encouragement to participate in exercises that will aid in strengthening muscles involved in their gait and the users medical professional will receive a set of charts that communicate metrics (such as the number of sit-to-stand exercises completed on the bench over sixty seconds). The metrics conveyed have been verified by physiologists as important indicators for determining deterioration in someones gait. If the health care professional notices a significant deterioration in the users performance they will be able to contact the user to alert them to seek consultation from their doctor.

\section{A. The Smart Bench}

After NFC tag on identifies the user for direction of data the user would be alerted with simple instructions of how to use the bench followed by an indication of where to find more detailed instructions and information about the system. A sixty second timer will start, during this time the aim of the user is to complete as many sit-to-stand exercise cycles as they can. Any measurement taken by the surface sensor's map or touch sensors will be recorded. This data is then read back and sent via HTTP Post with a custom API. The surface sensor map will provide data that can be used to measure the amount of sit-to-stand exercise the user has completed based on the amount of times the map is activated. It also provides data that can be used to determine where the user is landing and how heavily they are landing. A surface sensor mat at the foot will collect data that can be used to determine if the user is pushing hard with their left or right foot to stand and how even their balance is while standing. Capacitive touch sensors will be placed on a left and right handle as well as at the left and right of the front of the bench where users are liable to push off to help themselves stand. The purpose of these sensors is to understand if the user is requiring more or less aid to get up from a sitting position. Requiring upper body strength to aid in standing is known as an indicator to a lack of leg muscle strength. After the 60 second timer is complete the user will be alerted and can leave with no other action required.

\section{B. Smart Path}

The path concept uses very similar technology as the bench though in a different format. The user will have to tag on the NFC card at the beginning of the path. The entire length of the path is $7000 \mathrm{~mm}$ with $1000 \mathrm{~mm}$ space with no embedded sensor at the start and at the end of the track. The space at the start is needed so that the user will have time to get to their usual walking speed before the actual measurement is done. The $5000 \mathrm{~mm}$ active part of the path will be embedded with surface sensor. The surface sensor will aid in the collection of data that will be used to measure gait speed, step length, stride length and foot pressures. Time and distance are critical component in measuring the Gait Speed. We already know that the total distance that will be covered is $5000 \mathrm{~mm}$. However, this can be measured more accurately by taking into account the first foot contact and the last foot contact to the edge of the sensor. The start time will be derived from the first instance the user set 
foot on the sensor. The end time will be derived from the last recorded pressure. Stride length will be measured as distance between two successive placements of the same foot i.e. the length of two steps forward. The surface sensor is designed with the capability to identify the users foot placement in the path. The resolution will be enough to measure average foot pressures in order to print out a mapping of the specific pressure points. The comparison of foot pressure is important to detect any one sided weakness that could be caused by an onset of disease. In addition to the foot pressure, the path will also be equipped with handrails embedded with capacitive touch sensors. The surface sensor will detect if the user needed to use the handrail at any point during the walk and which side is being used more. There is no need to tag off at the end of the path. The system will be designed to detect the last foot step and will then store the measurements in the same way as the Bench exercise. The raw data will be sent to the database via HTTP Protocol.

\section{PRototypical System}

The current prototypical system is a working bench prototype that can fully communicate capacitive touch data and surface sensor data from the seat plate. The seat map provides enough data to determine the amount of sit-to-stand exercises that the user completes per session as well as data to determine where they are landing each time they sit and how hard they are landing on the seat. The test bench is constructed predominantly of Medium-density fibreboard and therefore is not yet water or weather proof, but is ready for initial user testing. The placement of sensors on the bench and on the path is shown in Figure 2.

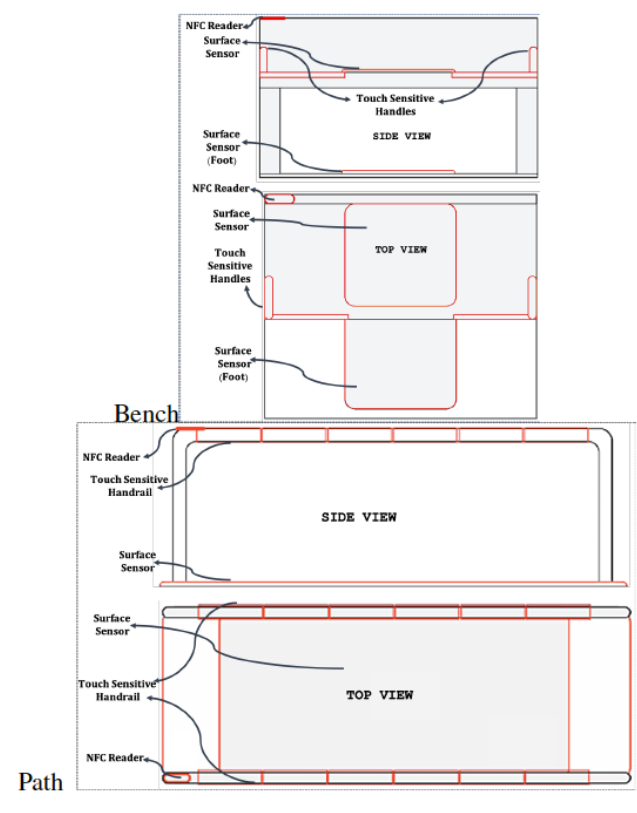

Fig. 2. Sensor placement.

The whole system is developed in four different stages that start from the sensors and ends with applications, to present useful information to the users.

\section{A. Stage 1: Sensors}

There are two sensors used for the current bench prototype - capacitive touch sensors and custom made pressure sensor. These sensors provide numerous derivatives that can offer insight into a persons gait. Due to pressure sensor's design, scalablity issues, and cost-benefit analysis, a new novel surface sensor was developed.The details of the sensor's development is discussed in detail in Section V. Also integrated are twelve key capacitive touch sensor also uses conductive material to supply charge to the sensor, when the capacitor reaches a certain level of charge (i.e. the conductive material is touched by a user) it triggers a process that records the timers millisecond count which is logged to an array of values belonging to each side of the bench.

\section{B. Stage 2: Data Aggregation}

Arduino serves as a microcontroller where the sensor data is collected and pre-processed from analog to digital formats. The Arduino, through a Wifi shield, is also responsible for sending the data to the internet. An HTTP protocol was used to communicate this data to the internet. Through the use of a RESTful API it was possible to send the data to a MySQL database which connects locally to the server on a Google Cloud virtual machine.

\section{Stage 3: Data Processing}

The data that was sent from Arduino to the MySQL database is still raw and will need to be analysed. A separate program written in Java handles this analysis. This Java application connects to the MySQL database through the RESTful API. It retrieves the raw data, applies algorithms and sends the output to the final database storage, Googles Firestore. Currently the process of running this application is done manually. In the future it could be executed inside the same virtual machine as the MySQL database and set it to run automatically on a scheduled basis.

\section{Stage 4: User Applications}

Through Google Cloud Firestore SDK we are able to connect both the Web application and Android application to the Firestore database. The applications can be accessed by users that have authorisation instantiated within Firebase. Users can query specific user data by searching their NHI through an HTML form. Both applications present user data through charts rendered using FusionCharts library.

\section{Surface Sensor Development}

As the system require a sensor that can be small enough to detect the multiple areas a foot exerts pressure and large enough to cover a $1 \mathrm{~m} \times 5 \mathrm{~m}$ pathway and have adequate scalability, repeatability. Many trails were conducted over a period of six months on various pressure, force sensors that are readily available in the market for integrating in to the system, in order to detect persons gait by the system. However, there are many issues related to the scalabily, repetablity and also rigidity of those sensors. In order to 
meet the system's requirements a novel surface sensor was developed, which is made out of velostat material. Velostat material is made out polymeric foil (polyolefins) impregnated with carbon black to make it electrically conductive. The material has the property of changing its resistance with either flexing or pressure, which is one of the key requirement in this application. During initial prototyping, copper strips of 2 $\mathrm{cm}$ width were glued in a matrix format on either side of the velostat material. Where each copper strip intersects creates a sensor point, this can be seen in Figure 3. Once the proof of concept has been established, an next prototype was designed using flexible Printed Circuit Board (PCB).

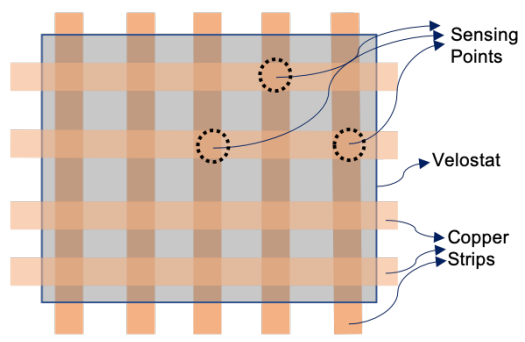

Fig. 3. Schematic of Surface Sensor

With this design, the resolution can easily be increased by increasing the number of copper strips. On one side of the material, a voltage will be passed through the copper and on the other side, the copper will be connected to a pull-down resistor creating a voltage divider. As the resistance of the velostat material changes when the pressure has applied the change in voltage can be measured to determine if pressure is applied or not. The part of the sensor that houses the copper strips need to be able to flex and compress when force is applied. Also the copper strips need to be placed known locations. This was achieved through milling a flexible PCB. The PCB as $25 \mathrm{~cm} \times 25 \mathrm{~cm}$ and consisted of thirty two $4 \mathrm{~mm}$ wide exposed copper strips. Each strip was placed $3.8 \mathrm{~mm}$ apart. The velostat material was then placed between two PCB's to make a $25 \mathrm{~cm} \times 25 \mathrm{~cm}$ sensor. As each PCB has 32 copper strips the sensor has 32 rows and 32 columns resulting in 1024 sensor points. The sensor mat can be seen in Figure 4.

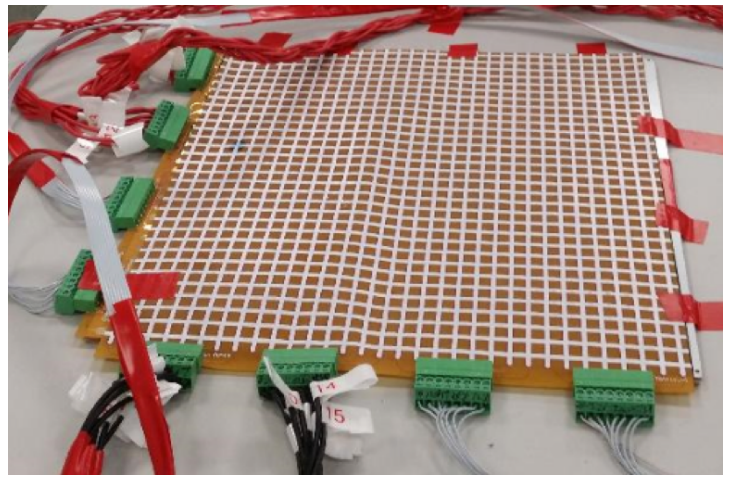

Fig. 4. Flexible PCB Based Surface Sensor

The Arduino Mega 2560 was used to read the power row within $1.3 \mathrm{~ms}$. As each power row consist of reading four shift registers, therefore each shift register takes approximately 325 s to read. The pMOS transistor takes 200 s to fully turn $\mathrm{ON}$, a delay of at least 450s would be needed before the shift register are read to ensure that all the shift registers data is read. The current into each pMOS was limited to $20 \mathrm{~mA}$ (the maximum supply current from the $16: 1$ multiplexer). 3 volts passes through the gate resistor, thus the current drawn from the $16: 1$ multiplexer is $12 \mathrm{~mA}$, as this is ideal maximum drawn current. With the added delay of 400s all sensor point are scanned within $48.4 \mathrm{~ms}$.

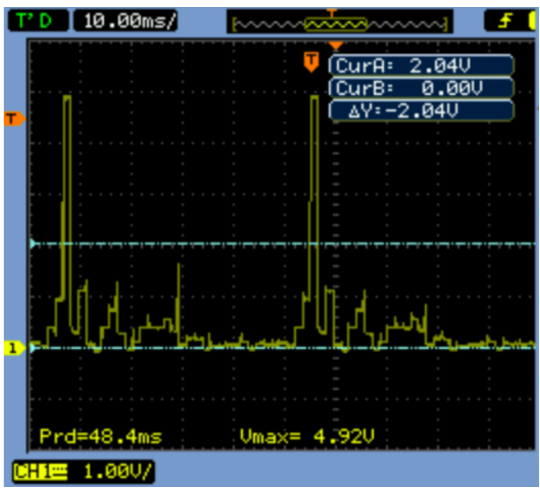

Fig. 5. Digital output of surface sensor mat

In Figure 5, shows the waveform of three adjacent rows, there is also noise present on each from the adjacent row, but this not an issue as the peak voltage of the noise is below $2 \mathrm{~V}$. The data is currently being displayed as matrix through the Arduinos serial communication port. Figure 6 shows the representation of data, where a "0" represents a non-activated point and "1" represents a activated point. This data is further processed to print out a mapping of the specific pressure points.

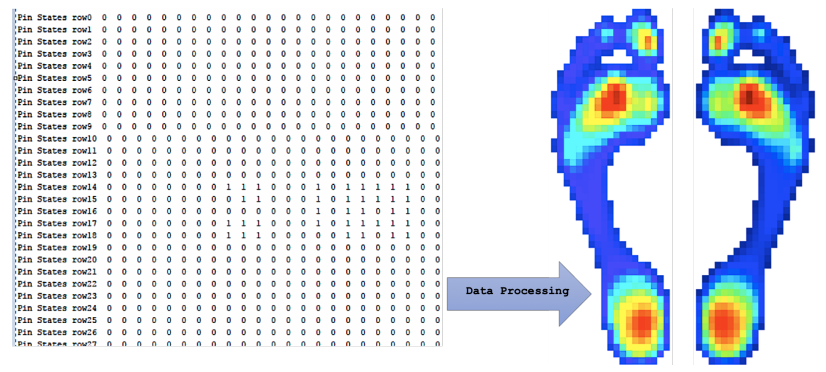

Fig. 6. Response of surface sensor mat

\section{DATA ANALYSIS}

Data has been a driving force behind this project, with an established exercise providing a medium for doctors, health care providers to gain an initial insight into a patients gait, the comparison of data gives us the ability to derive information from repeated exercises and build a stronger understanding of behaviour and patterns over time. There are two databases being used in the system: a MySQL database for raw data and Google Firestore for the clean data. 


\section{A. MySQL Database}

All sensor readings are stored as Strings and passed on to the MySQL database for storage. The database has two entities: session and feed. A session is one exercise event that the patient has performed. One feed is equivalent to one sensor reading. Each exercise session sends multiple sensor readings/feeds; hence the Entity Relationship diagram expressed that one session has many feeds.

\section{B. Google Firestore}

The data from MySQL database is accessed by the Java application that is responsible for processing and analysing the data. The output of this processing is then stored in Google Firestore. This data is then accessed by the Web application that is responsible for presenting the results in graphs. Google Firestore is a NoSQL database and the data design is based on the concept of documents and collection. For this system there is a collection called patients that contains all Patient documents. A Patient document contains a patients basic information as fields and two collections of clean data, PathCleanData and BenchCleanData. Each of these collections contains all of the clean data that is the output from the Java application.

\section{RESTful API}

The RESTful API is written in Node js using the Express framework. The API serves as a controller/middle-ware between the Arduino and the MySQL database and between MySQL database and the Java Application. Both the MySQL database and the RESTful API are running on a virtual machine hosted on the Google Cloud Platform. The API is accessible via its public external IP address and it connects to the MySQL database via a local-host port running inside the virtual machine. The API and the virtual machine are designed to run continually.

\section{Web Application}

The web application is designed in way that the healthcare professional can login using an authenticated email and password. Also, the healthcare professionals are able to search patients using the patients national health record number. Vue.js framework was used to build the Web app. It is a progressive JavaScript framework that uses components to render the web pages. The landing page on the web application will show a summary graph for both the bench and the path. For greater detail of the patients activity the healthcare professional can view the Bench or Path tabs which have subtabs with derivatives of the data collected from that patient.

\section{E. Mobile Application}

The mobile application is developed to render charts, information for the end user. The user can $\log$ on to the mobile application using a designated username and password, also the users can view basic metrics of their exercise history as shown in Figure 7.

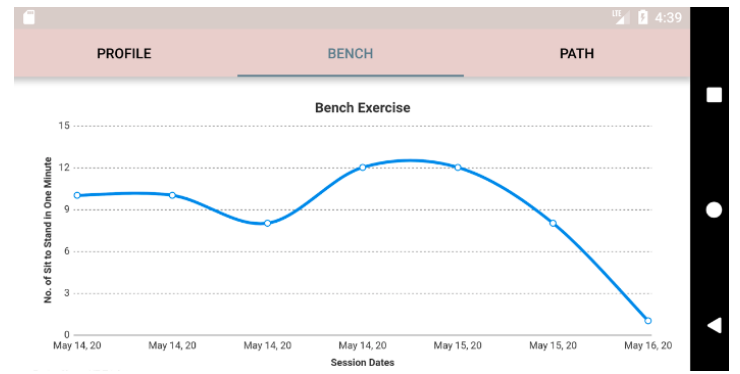

Fig. 7. Android app

\section{CONCLUSIONS}

This research has developed a proof of concept that can be applied to a larger scale when envisioning a public rehabilitation space. Though an immense amount of work would be required between now and establishing a public outdoor rehabilitation space it can be confirmed that the technology required for this concept is available and has the ability to interface to create the desired system. From a technical perspective, there are numerous functions that could be created and improved easily due to the thorough infrastructure implemented. With healthcare being an established right within many developed countries, it is in the interest of researchers to develop economical methods to guide and aid the public in receiving adequate health care. Detecting gait disorders earlier will allow for functional relief to the healthcare system with the possibility of reducing the number and severity of gait disorders through early intervention.

\section{REFERENCES}

[1] WHO, World report on ageing and health. World Health Organization, 2015.

[2] A. Kalache and K. Sen, "Ageing and health," in The ageing and development report. Routledge, 2017, pp. 77-88.

[3] O. Nee, A. Hein, T. Gorath, N. Hülsmann, G. B. Laleci, M. Yuksel, M. Olduz, I. Tasyurt, U. Orhan, A. Dogac et al., "Saphire: intelligent healthcare monitoring based on semantic interoperability platform: pilot applications," IET communications, vol. 2, no. 2, pp. 192-201, 2008.

[4] J. Tan, E-health care information systems: an introduction for students and professionals. John Wiley \& Sons, 2005.

[5] S. Merilampi and A. Sirkka, Introduction to Smart E-Health and E-Care Technologies. CRC Press, 2016.

[6] N. Suryadevara, A. Gaddam, R. Rayudu, and S. Mukhopadhyay, "Wireless sensors network based safe home to care elderly people: Behaviour detection," Sensors and Actuators A: Physical, vol. 186, pp. 277-283, 2012.

[7] C. Kershaw, J. McIntosh, B. Marques, J. Cornwall, L. Stoner, and P. Wood, "A potential role for outdoor, interactive spaces as a healthcare intervention for older persons," 2017.

[8] L. Cranney, P. Phongsavan, M. Kariuki, V. Stride, A. Scott, M. Hua, and A. Bauman, "Impact of an outdoor gym on park users physical activity: A natural experiment," Health \& place, vol. 37, pp. 26-34, 2016.

[9] A. R. Othman and F. Fadzil, "Influence of outdoor space to the elderly wellbeing in a typical care centre," Procedia-Social and Behavioral Sciences, vol. 170, pp. 320-329, 2015.

[10] M. Hemmatpour, M. Karimshoushtari, R. Ferrero, B. Montrucchio, M. Rebaudengo, and C. Novara, "Polynomial classification model for real-time fall prediction system," in Proc. IEEE 41st Annual Computer Software and Applications Conf. (COMPSAC), vol. 1, Jul. 2017, pp. 973-978. 
[11] N. K. Suryadevara, A. Gaddam, S. C. Mukhopadhyay, and R. K. Rayudu, "Wellness determination of inhabitant based on daily activity behaviour in real-time monitoring using sensor networks," in Proc. Fifth Int. Conf. Sensing Technology, Nov. 2011, pp. 474-481.

[12] A. Gaddam, S. C. Mukhopadhyay, and G. S. Gupta, "Elder care based on cognitive sensor network," IEEE Sensors Journal, vol. 11, no. 3, pp. 574-581, March 2011.

[13] C. Wang, X. Wu, N. Li, and Y. L. Chen, "Abnormal detection based on gait analysis," in Proc. 10th World Congress Intelligent Control and Automation, Jul. 2012, pp. 4859-4864.

[14] P. Soda, A. Carta, D. Formica, and E. Guglielmelli, "A low-cost videobased tool for clinical gait analysis," in Proc. Annual Int. Conf. of the IEEE Engineering in Medicine and Biology Society, Sep. 2009, pp. 3979-3982.

[15] T. Heikkil, E. Strmmer, S. Kivikunnas, M. Jrviluoma, M. Korkalainen, V. Kyllnen, E. M. Sarjanoja, and I. Peltomaa, "Low intrusive ehealth monitoring: human posture and activity level detection with an intelligent furniture network," IEEE Wireless Communications, vol. 20, no. 4, pp. 57-63, Aug. 2013.

[16] M. Chan, E. Campo, D. Brulin, and D. Esteve, "Biomedical monitoring technologies and future healthcare systems," 2017.

[17] Y. Cedervall, K. Halvorsen, and A. C. berg, "A longitudinal study of gait function and characteristics of gait disturbance in individuals with Alzheimer's disease," Gait \& Posture, vol. 39, no. 4, pp. 1022-1027, Apr. 2014. [Online]. Available: http://dx.doi.org/10.1016/j.gaitpost.2013.12.026

[18] M. Balazia and K. N. Plataniotis, "Human gait recognition from motion capture data in signature poses," IET Biometrics, vol. 6, no. 2, pp. 129 $137,2017$.

[19] T. Sugiyama and C. W. Thompson, "Outdoor environments, activity and the well-being of older people: conceptualising environmental support," Environment and Planning A, vol. 39, no. 8, pp. 1943-1960, 2007.

[20] R. Leiros-Rodríguez and J. L. García-Soidan, "Balance training in elderly women using public parks," Journal of women \& aging, vol. 26, no. 3, pp. 207-218, 2014.

[21] D. v. Kooten, F. Hettinga, K. Duffy, J. Jackson, and M. J. D. Taylor, "Are there associations with age and sex in walking stability in healthy older adults?" Gait \& Posture, vol. 60, pp. 65 - 70, 2018. [Online]. Available: http://www.sciencedirect.com/science/article/pii/S0966636217310093

[22] R. Steele, A. Lo, C. Secombe, and Y. K. Wong, "Elderly persons perception and acceptance of using wireless sensor networks to assist healthcare," International journal of medical informatics, vol. 78, no. 12, pp. 788-801, 2009.

[23] Y. Cedervall, K. Halvorsen, and A. C. Åberg, "A longitudinal study of gait function and characteristics of gait disturbance in individuals with alzheimer's disease," Gait \& posture, vol. 39, no. 4, pp. 1022-1027, 2014.

[24] M. Cesari, S. B. Kritchevsky, B. W. Penninx, B. J. Nicklas, E. M. Simonsick, A. B. Newman, F. A. Tylavsky, J. S. Brach, S. Satterfield, D. C. Bauer et al., "Prognostic value of usual gait speed in well-functioning older peopleresults from the health, aging and body composition study," Journal of the American Geriatrics Society, vol. 53, no. 10, pp. 1675$1680,2005$.

[25] A. Öztürk, T. T. Şimşek, E. T. Yümin, M. Sertel, and M. Yümin, "The relationship between physical, functional capacity and quality of life (qol) among elderly people with a chronic disease," Archives of Gerontology and Geriatrics, vol. 53, no. 3, pp. 278-283, 2011.

[26] D. van Kooten, F. Hettinga, K. Duffy, J. Jackson, and M. J. Taylor, "Are there associations with age and sex in walking stability in healthy older adults?" Gait \& posture, vol. 60, pp. 65-70, 2018. 